\title{
A non-radioactive mass spectrometry based differential radial capillary action of ligand assay (DRaCALA) to assess ligand binding to proteins
}

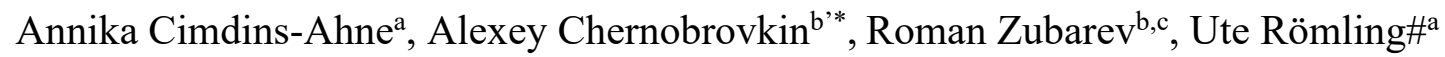

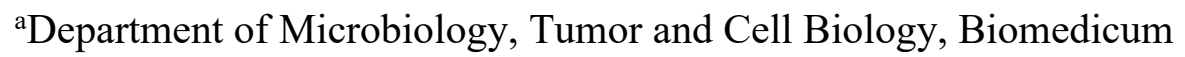

Solnavägen 9, 17165 Solna, Sweden

${ }^{b}$ Medical Biochemistry and Biophysics, Karolinska Institutet, Biomedicum, Solnavägen 9, 17165 Solna, Sweden

${ }^{\mathrm{c}}$ Department of Pharmacological \& Technological Chemistry, I.M. Sechenov First Moscow State Medical University, Moscow, 119146, Russia

*current address: Pelago Bioscience AB, Banvektsvägen 20, 17148 Solna, Sweden

\# Corresponding author: ute.romling@ki.se

subject category: Mass spectrometry; Technical Note

keywords: biofilm, cyclic di-GMP, non-radioactive DRaCALA, mass spectrometry, YciR, BcsE

Abbreviations used: cyclic di-GMP, bis- $\left(3^{\prime}-5^{\prime}\right)$-cyclic dimeric guanosine monophosphate; cyclic di-AMP, bis-( $\left.3^{\prime}-5^{\prime}\right)$-cyclic dimeric adenosine monophosphate; MALDI-TOF MS, matrix-assisted laser desorption and ionization time-of-flight mass spectrometry; DRaCALA, differential radial capillary action of ligand assay; GGDEF motif, diguanylate cyclase motif; EAL motif, phosphodiesterase motif 
Graphical abstract

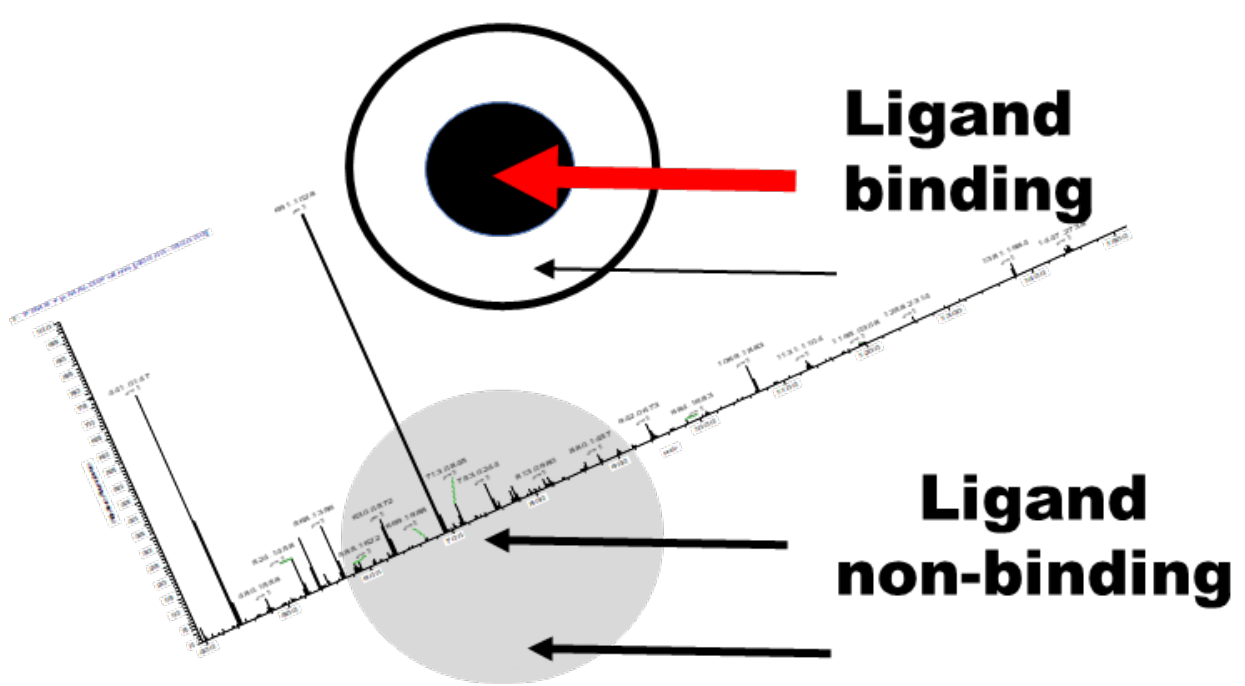




\section{Highlights}

- Cyclic di-nucleotides are ubiquitous second messengers in Bacteria, however, few receptors have been identified.

- Previous screening of cell lysates by differential radial capillary action of ligand assay (DRaCALA) using radioactive ligand identified cyclic dinucleotide binding proteins.

- A MALDI-TOF based DRaCALA was developed to detect cyclic dinucleotide binding as a non-radioactive alternative.

- Known cyclic di-GMP binding proteins were verified and potential cyclic diGMP binding proteins were identified. 


\begin{abstract}
Binding of ligands to macromolecules changes their physicochemical characteristics. Cyclic di-GMP and other cyclic di-nucleotides are second messengers involved in motility/sessility and acute/chronic infection life style transition. Although the GGDEF domain encoding preferentially a diguanylate cyclase represents one of the most abundant bacterial domain superfamilies, the number of cyclic di-GMP receptors falls short. To facilitate screening for cyclic di-nucleotide binding proteins, we describe a non-radioactive, MALDI-TOF based modification of the widely applied differential radial capillary action of ligand assay (DRaCALA). The results of this assay suggest that $\mathrm{YciR}_{\mathrm{Fec} 101}$, but not the $\mathrm{YciR}_{\mathrm{TOB} 1}$ variant of the diguanylate cyclase/phosphodiesterase YciR binds cyclic di-GMP.
\end{abstract}




\section{Introduction}

Binding of small molecules to macromolecules such as proteins has a deep physiological impact as it alters the physico-chemical properties and the functionality of the protein including alteration of enzymatic parameters through allosteric regulation and change in protein-protein interactions due to substantial conformational changes upon small molecule binding [1].

There are a variety of directed and unbiased experimental approaches to determine binding of small molecules to proteins and to assess their binding affinity [2]. Traditional biochemical approaches require purified protein and are not highthroughput. One of the most successful experimental approaches suitable for screening of a large number of candidate receptors for binding with candidate small ligand is the differential radial capillary action of ligand assay (DRaCALA), which does not require purification of the expressed protein, but uses whole bacterial cell lysates containing plasmid-expressed candidate gene products [3-6]. Thereby, small ligand binding to a protein is assessed by the spot intensity and diameter upon spotting the protein extract containing ligand onto a protein-binding nitrocellulose membrane. Binding of the radioactive ligand to the macromolecule creates a small intensive spot reflecting the protein location, while, upon non-binding, the ligand signal is more extended, but overall equally intensive reflecting the free ligand distribution over a larger spot area created by capillary forces.

Cyclic di-nucleotides are ubiquitous second messengers in Bacteria, however, the abundance of cyclic di-GMP turnover proteins is still not reflected by the number of identified receptors [7]. DRaCALA has been frequently used to identify receptors for cyclic di-nucleotides, in particular cyclic di-GMP and cyclic di-AMP, which are ubiquitous second messengers in Gram-negative and Gram-positive Bacteria [8,9]. Thereby lysates of bacterial libraries containing all plasmid-expressed gene products of an organism have been screened for cyclic di-nucleotide binding proteins. With this experimental approach, receptors involved in, for example, regulation of cellulose biosynthesis such as BcsE and maintenance of osmohomeostasis and potassium transport have been identified $[4,10]$.

A radioactive ligand, as in the originally described approach, needs to be prepared and labeling is not necessarily readily applicable to every small molecular compound. However, MALDI-TOF MS or imaging mass spectrometry can be used to detect binding of a ligand to a protein receptor on the nitrocellulose membrane. Thereby, assessment of binding can be achieved by measuring the ligand concentration at the application spot and at the edge of the larger spot area that the liquid reached by capillary action (Fig. 1).

To perform this assay, a single colony of the E. coli strains (in case of the control with the vector containing a cloned cyclic di-GMP binding protein ( $\mathrm{YcgR}$ or $\mathrm{BcsE}$ ) and its relevant non-binding mutant) were inoculated 1:100 from an overnight culture in LB medium with the relevant antibiotic. After induction with $0.1 \% \mathrm{~L}$-arabinose at $30^{\circ} \mathrm{C}$ for four hours, cells were harvested by resuspension in binding buffer $(10 \mathrm{mM}$ Tris pH 8.0, $100 \mathrm{mM} \mathrm{NaCl}, 5 \mathrm{mM} \mathrm{MgCl}_{2}$, in Chromasolv $\mathrm{H}_{2} \mathrm{O}$; [11] with $100 \mu 1$ lysozyme $(500 \mu \mathrm{g} / \mathrm{ml})$ per $\mathrm{OD}_{600}=3([4,12])$. Cells were disrupted by two freeze-thaw cycles of $1 \mathrm{~h}$ at room temperature and at $-80^{\circ} \mathrm{C} .18 \mu \mathrm{l}$ cell lysate and $2 \mu 110 \mu \mathrm{M}$ c-di-GMP were mixed, incubated $10 \mathrm{~min}$ at room temperature and $2 \mu \mathrm{l}$ were spotted on a nitrocellulose membrane (Schleicher \& Schuell Protan ${ }^{\mathrm{TM}}$, BA85). After the membrane had completely dryed, equally sized membrane pieces at the application spot and the edge of the diffused liquid were cut out with a scalpel. $2 \mu 15 \mathrm{mg} / \mathrm{ml}$ alpha-cyano-4-hydroxy-cinnamic acid matrix in 50\% acetonitrile and $0.05 \%$ 
triflouroacetic acid were applied on a MALDI MassTech 96 spot sample plate, followed by the membrane piece and again $2 \mu 1$ matrix. The matrix was left to dry at room temperature, the membrane removed and the sample measured in a QExactive, Thermo Scientific, with a MassTech AP MALDI PDF Ion source. Settings of the MALDI source were sheath gas: 0 ; aux gas: 0 ; sweep gas: 0 ; spray voltage: $3,75 \mathrm{kV}$; spray current $[\mu \mathrm{A}]$; capillary temperature: 280; S-lens RF level: $100.0 ; 5 \mathrm{~min}$ acquisition time, 2 microscans; lock masses OFF; $1 \mathrm{e}^{5}$ AGC target; maximal inject time 1.000. Signals for cyclic di-GMP were observed as expected at around 691.1 $[\mathrm{M}+\mathrm{H}]^{+}$and $713.1[\mathrm{M}+\mathrm{Na}]^{+}$.

In order to validate the procedure, we first expressed two previously characterized cyclic di-GMP binding proteins and their respective binding mutants, namely the cellulose biosynthesis enhancer BcsE and its non-binding mutant $B c s E_{R 415 D}$ and the flagella motor 'backstop-break' protein YcgR and its nonbinding mutant $Y_{\operatorname{cgR}} R_{\mathrm{R} 18 \mathrm{D}}$ in E. coli Top 10 [4, 13]. Measurement of the cyclic di-GMP counts from cell lysates expressing these receptors after incubation with cyclic di-GMP showed higher counts derived from the membrane taken from the application spot than from the periphery. In contrast, counts were similar when membranes from cell lysates expressing the non-binding protein variants were investigated after incubation with cyclic di-GMP (Fig. 2 a).

YciR, an enzyme with opposing diguanylate cyclase and phosphodiesterase activities, has been initially characterized to downregulate expression of the agar-grown rdar biofilm morphotype in Escherichia coli and Salmonella typhimurium by a mechanism independent of its catalytic activities involving cyclic di-GMP sensing and proteinprotein interactions [14-17]. Natural YciR protein variants encoded by semiconstitutive rdar biofilm strains, though, failed to efficiently downregulate this morphotype, even when overexpressed. Sensing and binding of cyclic di-GMP might be a determinative factor for the different variant behavior. To assess whether two YciR protein variants differentially bind cyclic di-GMP and to assess their binding capacity, we choose to express the two variants of the opposing activity enzyme YciR, YciR ${ }_{\mathrm{Fec} 101}$, that has been previously shown to downregulate the rdar morphotype, and $\mathrm{YciR}_{\mathrm{TOB} 1}$ that is nearly invariant to rdar morphotype expression upon plasmid-based expression [13]. Upon expression of YciR from the commensal E. coli strain Fec101, the MALDI-TOF MS -based DRaCALA binding assay showed higher counts for the membrane derived from the application spot compared to the membrane from the periphery suggesting binding of cyclic di-GMP to $\mathrm{YciR}_{\mathrm{Fec} 101}$. In contrast, membranes from the application spot and the periphery of the membrane for the $\mathrm{YciR}_{\mathrm{Fec} 101-\mathrm{K} 581 \mathrm{~A}}$ mutant and a YciR double mutant in the GGDEF and EAL motif, $\mathrm{YciR}_{\mathrm{Fec} 101-\mathrm{D} 316 \mathrm{~A}, \mathrm{E} 317 \mathrm{~A} / \mathrm{E} 440 \mathrm{~A} \text {, abolishing the two catalytic activities of the combined }}$ diguanylate cyclase and phosphodiesterase, showed similar counts suggesting those protein variants are either non-binding or not being expressed under the respective assay conditions. Equally, the counts from the application spot and the membrane periphery for $\mathrm{YciR}_{\mathrm{TOB} 1}$ were even higher for the periphery suggesting that $\mathrm{YciR}_{\mathrm{TOB} 1}$ does not bind cyclic di-GMP (Fig. 2b).

In conclusion, a MALDI-TOF MS-based DRaCALA assay to identify cyclic dinucleotide binding proteins was successfully implemented which should save experiment time, as production of radioactive cyclic di-GMP is not necessary. Future miniaturization of the experiment will save material and optimization of the choice of the protein-binding matrix with optimal capillary forces for small compound diffusion will optimize the detection process. Furthermore, the approach can be transferred to 
measure binding of other small ligands and molecules where methodological restrictions do not allow read radioactive labelling.

\section{Author contribution}

UR had the idea; ACA, RZ, AC and UR conceptualized the study; ACA and AC performed the experiments; ACA, RZ, AC and UR analyzed the data; UR and ACA wrote the manuscript with the input of all authors.

\section{Acknowledgement}

AC received a post-doctoral scholarship from the German Research Foundation (CI 239/1-1 and 239/2-1). This work was supported by the Swedish Research Council for Natural Sciences and Engineering (621-2013-4809) and the Karolinska Institutet. 


\section{Figures}
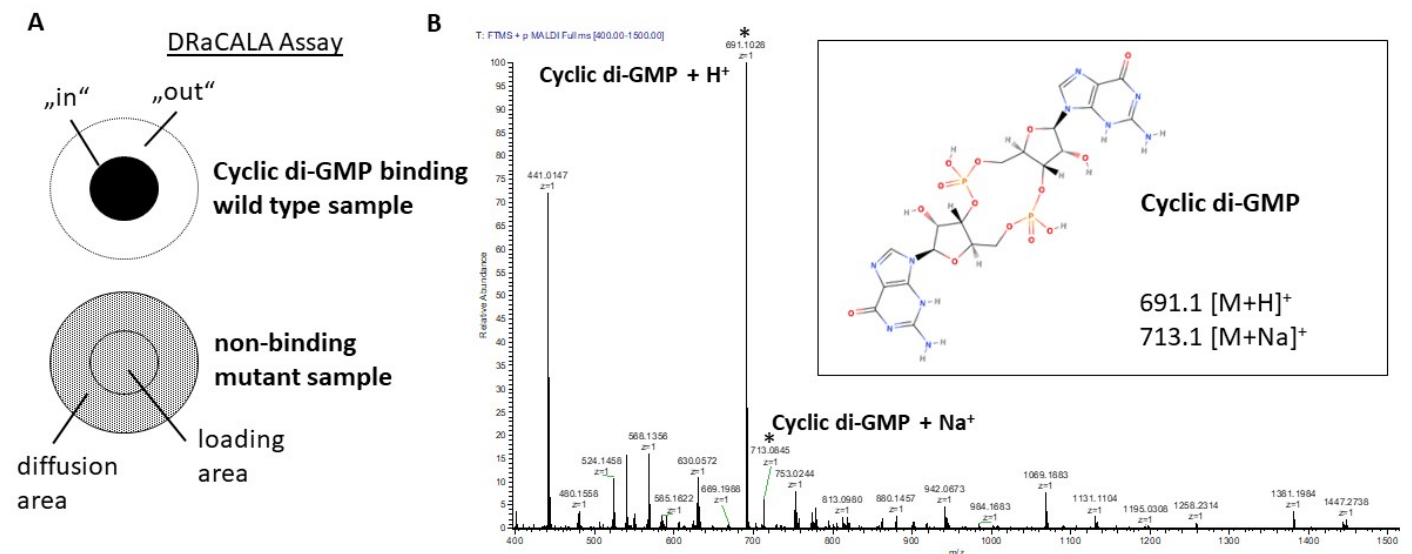

Figure 1: Basic principles of the non-radioactive, MALDI-TOF MS based DRaCALA assay

A Schematic drawing of the read out of the DRaCALA assay. If ligand is bound to the protein sample, the sample remains in the loading area while unbound ligand will diffuse $[2,4]$. Resulting ligand concentrations in the inner part of the loading spot (,in“) and peripheral location (,out") can subsequently be determined by quantitative mass spectrometry.

B Mass spectrum for cyclic di-GMP (Biolog), m/z 400-1500, main peak corresponds to positively charged protonated cyclic di-GMP (m/z 691.1). The inserted box depicts the structure of cyclic di-GMP and shows the molecular mass of the positively charged cyclic di-GMP molecules $\left([\mathrm{M}+\mathrm{H}]^{+},[\mathrm{M}+\mathrm{Na}]^{+}\right)$.

A

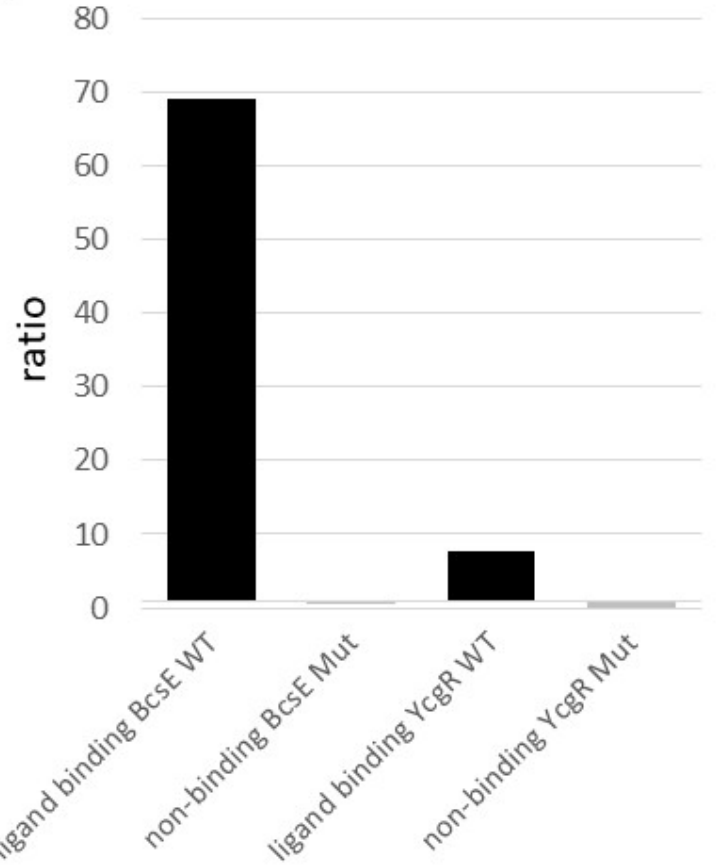

B

$$
25
$$

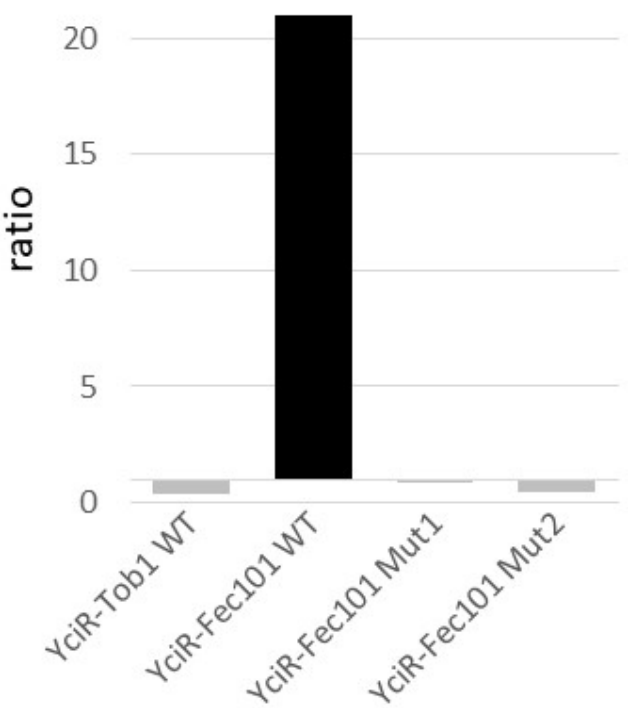

Figure 2: Determination of cyclic di-GMP binding capability of cyclic di-GMP receptors and candidate receptor proteins. 
Ratio of counts derived from representative absolute values measured by FTMS MALDI-TOF on the membrane at the application spot and the peripheral membrane (range $\mathrm{m} / \mathrm{z} 691.0950-691.1050$ ). A value $>1$ is indicative for cyclic di-GMP binding to the expressed protein (black bars), a value $<1$ indicates no binding (gray bars). A Proof of principle shown with the known cyclic di-GMP receptors BcsE and YcgR and their respective non-binding catalytic mutants $[3,12]$. B Cyclic di-GMP binding capabilities of the cyclic di-GMP metabolizing protein YciR from the commensal $E$. coli strains TOB1 and Fec101. While YciR Fec101 binds c-di-GMP, the respective catalytic mutants and $\mathrm{YciR}_{\mathrm{TOB} 1}$ do not. 


\section{References}

[1] S.L. Schreiber, Small molecules: the missing link in the central dogma, Nature chemical biology, 1 (2005) 64-66.

[2] A. McFedries, A. Schwaid, A. Saghatelian, Methods for the elucidation of proteinsmall molecule interactions, Chemistry \& biology, 20 (2013) 667-673.

[3] K.G. Roelofs, J. Wang, H.O. Sintim, V.T. Lee, Differential radial capillary action of ligand assay for high-throughput detection of protein-metabolite interactions, Proc Natl Acad Sci U S A, 108 (2011) 15528-15533.

[4] X. Fang, I. Ahmad, A. Blanka, M. Schottkowski, A. Cimdins, M.Y. Galperin, U. Romling, M. Gomelsky, GIL, a new c-di-GMP-binding protein domain involved in regulation of cellulose synthesis in enterobacteria, Molecular microbiology, 93 (2014) 439-452.

[5] V.T. Lee, Detection of Cyclic DInucleotide Binding Proteins, in: S.-H. Chou, Guiliani, N., Lee, V.T., Römling, U. (Ed.) Microbial Cyclic Di-Nucleotide Signaling, Springer, Cham, Switzerland, 2020, pp. 107-126.

[6] J. Yang, B.W. Anderson, A. Turdiev, H. Turdiev, D.M. Stevenson, D. AmadorNoguez, V.T. Lee, J.D. Wang, The nucleotide pGpp acts as a third alarmone in Bacillus, with functions distinct from those of (p) ppGpp, Nature communications, 11 (2020) 5388.

[7] U. Romling, M.Y. Galperin, M. Gomelsky, Cyclic di-GMP: the first 25 years of a universal bacterial second messenger, Microbiology and molecular biology reviews : MMBR, 77 (2013) 1-52.

[8] G. Witte, S. Hartung, K. Buttner, K.P. Hopfner, Structural biochemistry of a bacterial checkpoint protein reveals diadenylate cyclase activity regulated by DNA recombination intermediates, Mol Cell, 30 (2008) 167-178.

[9] T. Schirmer, U. Jenal, Structural and mechanistic determinants of c-di-GMP signalling, Nat Rev Microbiol, 7 (2009) 724-735.

[10] R.M. Corrigan, I. Campeotto, T. Jeganathan, K.G. Roelofs, V.T. Lee, A. Gründling, Systematic identification of conserved bacterial c-di-AMP receptor proteins, Proc Natl Acad Sci U S A, 110 (2013) 9084-9089.

[11] O.J. Lieberman, M.W. Orr, Y. Wang, V.T. Lee, High-throughput screening using the differential radial capillary action of ligand assay identifies ebselen as an inhibitor of diguanylate cyclases, ACS chemical biology, 9 (2014) 183-192.

[12] J.A. Moscoso, H. Schramke, Y. Zhang, T. Tosi, A. Dehbi, K. Jung, A. Gründling, Binding of Cyclic Di-AMP to the Staphylococcus aureus Sensor Kinase KdpD Occurs via the Universal Stress Protein Domain and Downregulates the Expression of the Kdp Potassium Transporter, J Bacteriol, 198 (2016) 98-110.

[13] D.A. Ryjenkov, R. Simm, U. Römling, M. Gomelsky, The PilZ domain is a receptor for the second messenger c-di-GMP. The PilZ domain protein YcgR controls motility in enterobacteria, J Biol Chem, 281 (2006) 30310-30314.

[14] A. Cimdins, R. Simm, F. Li, P. Lüthje, K. Thorell, A. Sjöling, A. Brauner, U. Römling, Alterations of c-di-GMP turnover proteins modulate semi-constitutive rdar biofilm formation in commensal and uropathogenic Escherichia coli, MicrobiologyOpen, 6 (2017).

[15] S. Lindenberg, G. Klauck, C. Pesavento, E. Klauck, R. Hengge, The EAL domain protein YciR acts as a trigger enzyme in a c-di-GMP signalling cascade in E. coli biofilm control, The EMBO journal, 32 (2013) 2001-2014.

[16] C. Yang, C. Cui, Q. Ye, J. Kan, S. Fu, S. Song, Y. Huang, F. He, L.H. Zhang, Y. Jia, Y.G. Gao, C.S. Harwood, Y. Deng, Burkholderia cenocepacia integrates cis-2- 
dodecenoic acid and cyclic dimeric guanosine monophosphate signals to control virulence, Proc Natl Acad Sci U S A, 114 (2017) 13006-13011.

[17] I. Ahmad, A. Cimdins, T. Beske, U. Römling, Detailed analysis of c-di-GMP mediated regulation of $\operatorname{csg} D$ expression in Salmonella typhimurium, BMC microbiology, 17 (2017) 27. 